\title{
AN ALGORITHM FOR THE ANALYSIS OF SEISMIC BASE ISOLATED SHEAR FRAME STRUCTURES
}

\section{Mohammad Ahmad Al-Samara}

Professor, Dean of the Higher Institute for Earthquake Studies \& Research, Damascus University, Syria

Thaer Mohammad Nael Trisi, M.Sc. Student, Damascus University

(Received October 10, 2010 Accepted Novemberber1, 2010)

In this research the governing relationships of the behavior of seismic isolated shear frame structures are presented and algorithm solution was written for multi degrees of freedom "MDOF" model, considering the masses lumping at the center of diaphragm in each story and taking into consideration the bilinear model of the isolators. To verify the algorithm a computer program was written using "Visual Basic" and a numerical example was solved. The results are compared with those obtained by using "SAP 2000” Program.

KEYWORDS: Seismic Base Isolation, Bilinear model, Rubber Isolators, Damping
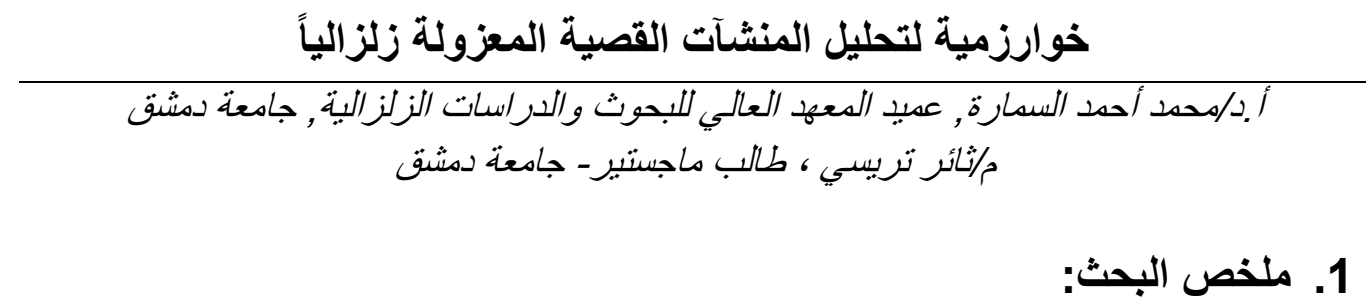

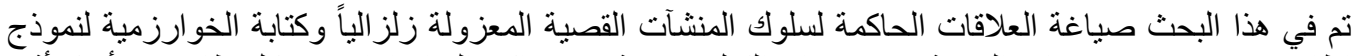

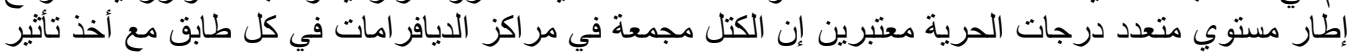

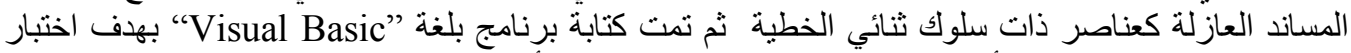

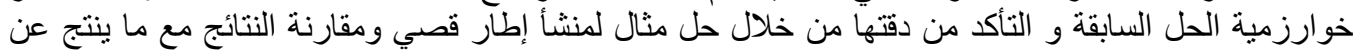

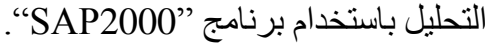
مفاتيح الكلمات: العزل الزلز الي, سلوك ثنائي الخطية, مساند عزل مطاطية, تخامد.

2. مقدمة:

يعتمد مبدأ العزل الزلزالي على جعل المنشأ خارج نطاق الخطر الناتج عن الهزات الأرضية وذلك بتقليل القوة

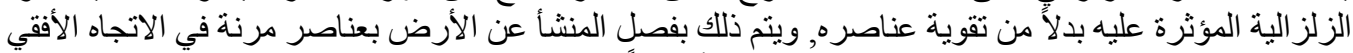

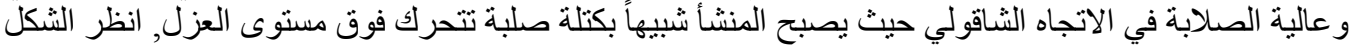
رقم (1).

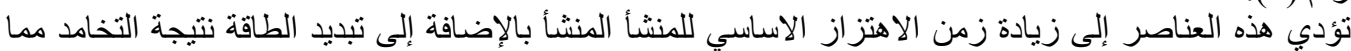
يحد من مقدار القوة الزلز الية وبالتالي إلى تخفيف الضرد الإني الذي تتعرض لله العناصر الإنشائية وغير الإنشائية. 


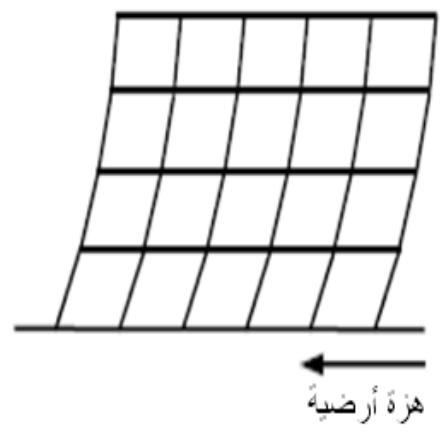

(a)

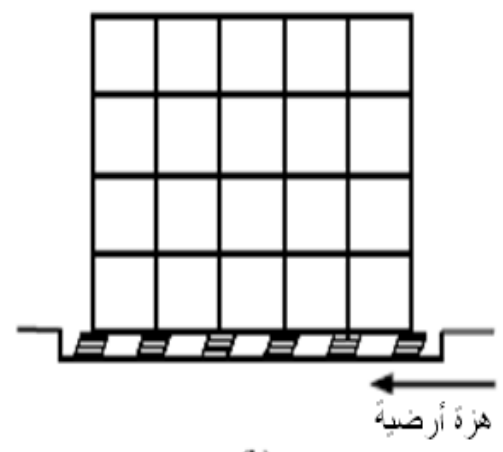

(b)

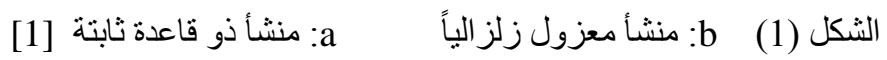

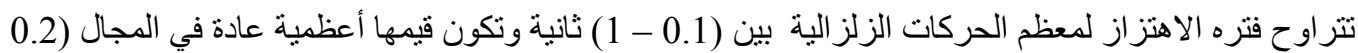

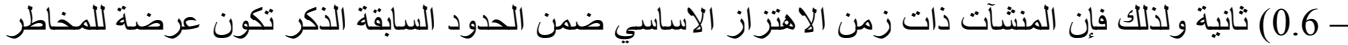

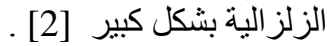
إن الخاصية الأكثر أهمية في المنشآت المعزولة زلز الياً هي زيادة المرونة في الاتجاه الأفقي مما يؤدي إلى زيادية

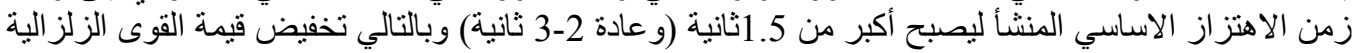
المطبقة عليه كما هو موضح في الثنكل (2)

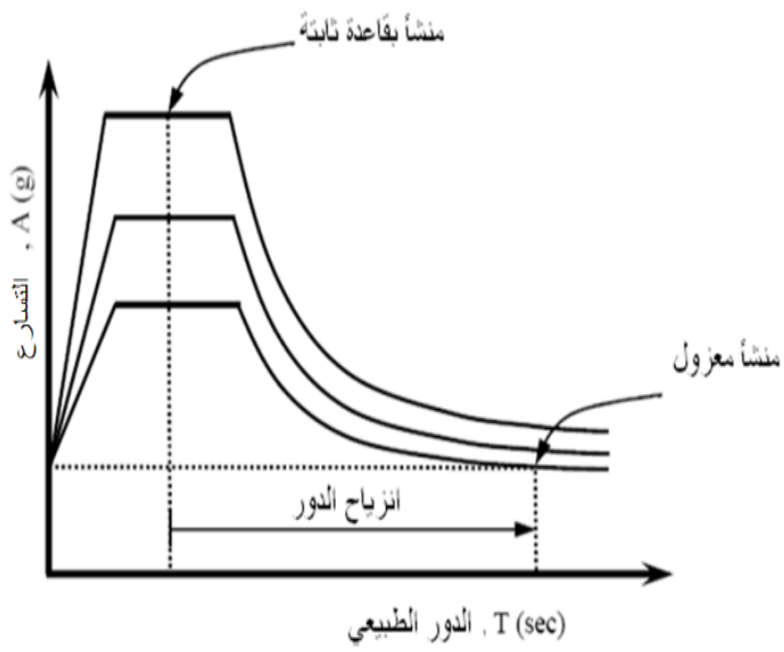

الثكل (2): تأثير زيادة زمن الاهتزاز الاساسي على التسارع الزلز الي [1]

تتراوح نسبة التخامد في المنشآت التقليدية حول(5\%) و وهي ناتجة عن التشوهات الحاصلة في العناصر الإنشائية

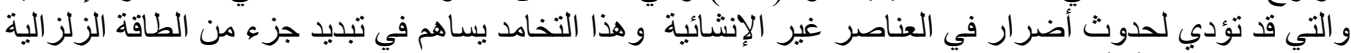

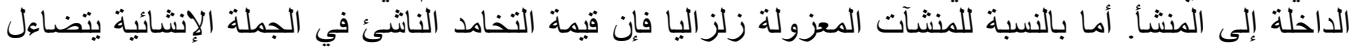

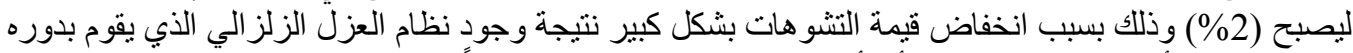

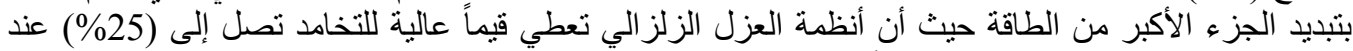

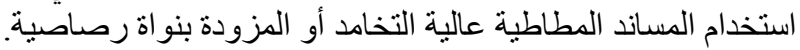
يوضح الثكل (3) نأثير ازدياد قيمة التخامد على منحني طيف الاستجابة حيث يمكن ملاحظة انخفاض قيمة

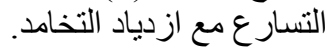




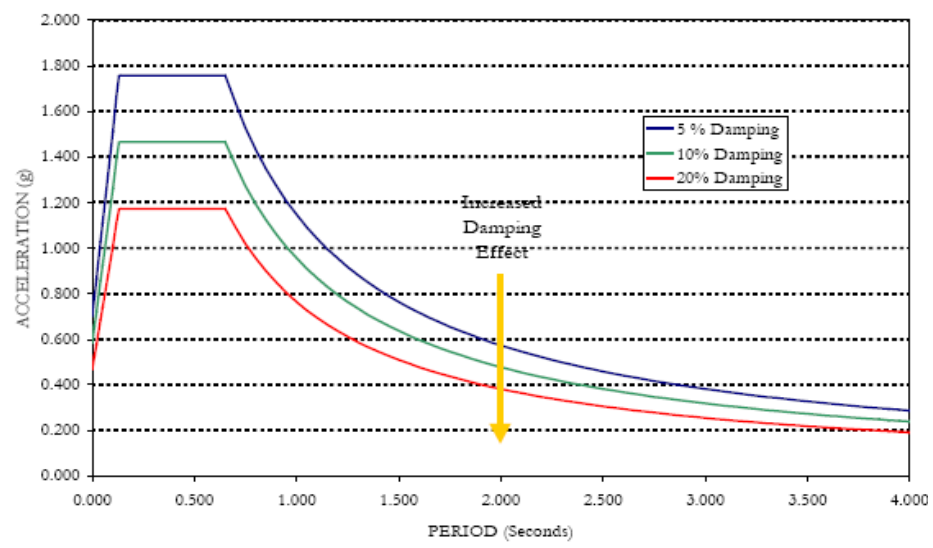

الثكل (3): تأثثير زيادة التخامد على منحني طيف الاستجابة [3]

قام الباحثان Masaru Kikuchi , Ian D.Aiken (1997) [4 نموذج تحليلي ترددي هستيري لمساند العزل

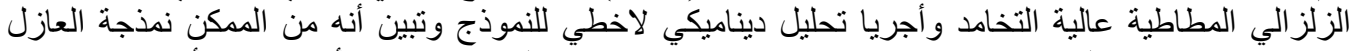

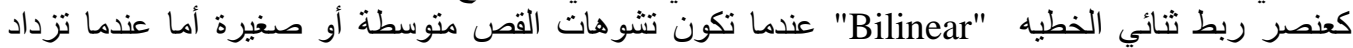

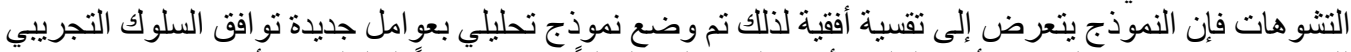

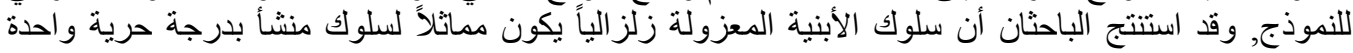
عندما تكون الجملة الإنشائية ذات صلان آنابة عالية.

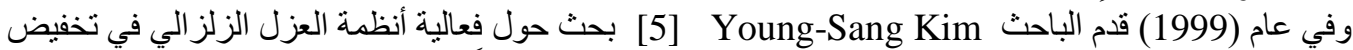

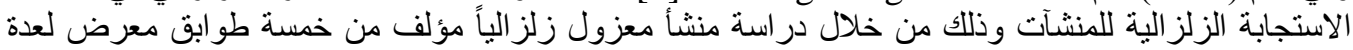

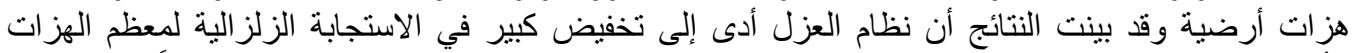

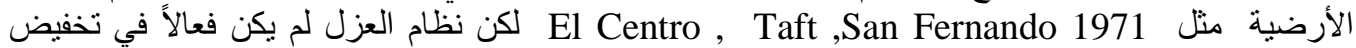

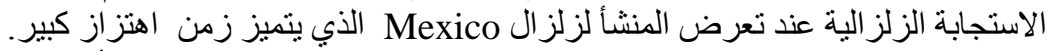

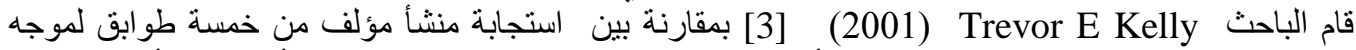

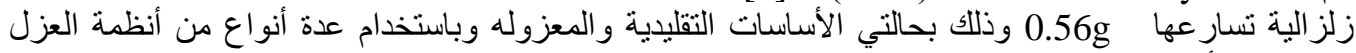

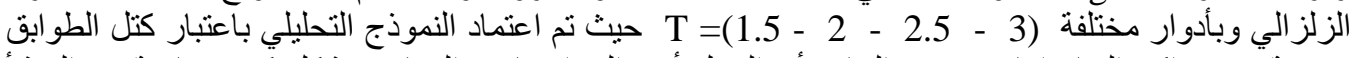

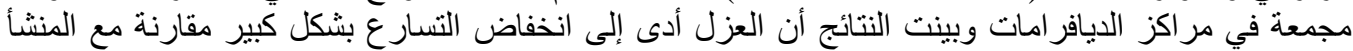

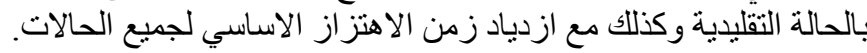

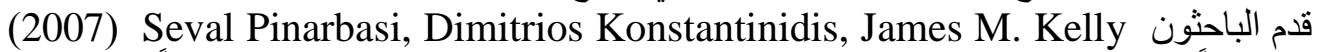

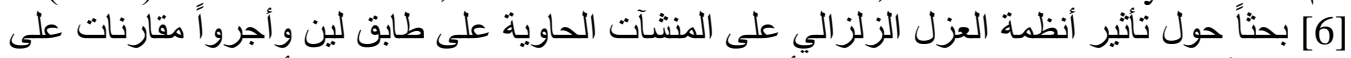

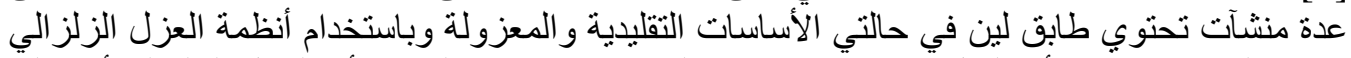

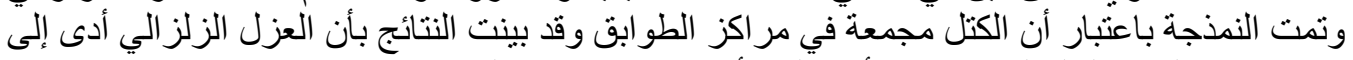

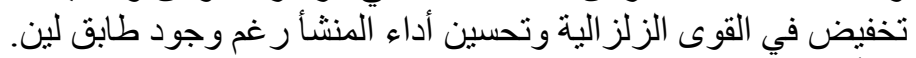
من أهم المساند المستخدمة في عزل المنشآت هي المساند المطاطية الطبيعية أو عالية التخامد أو المزودة بنواة رصاصية.

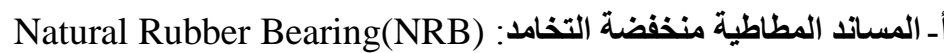
ينكون طرفاها العلوي و السفلي من صفائح فو لاذية سميكة إضافة إلى حشو ات من صفائح فو لاذية لاذية رقيقة توجد بينها

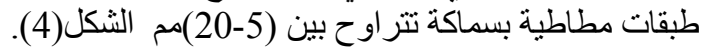

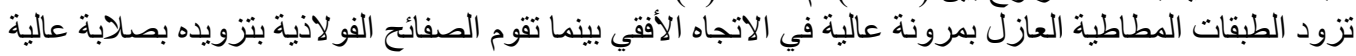

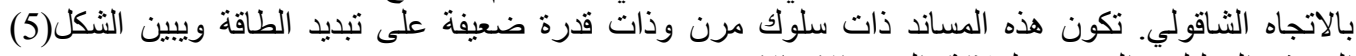

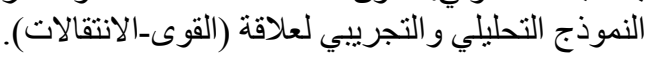



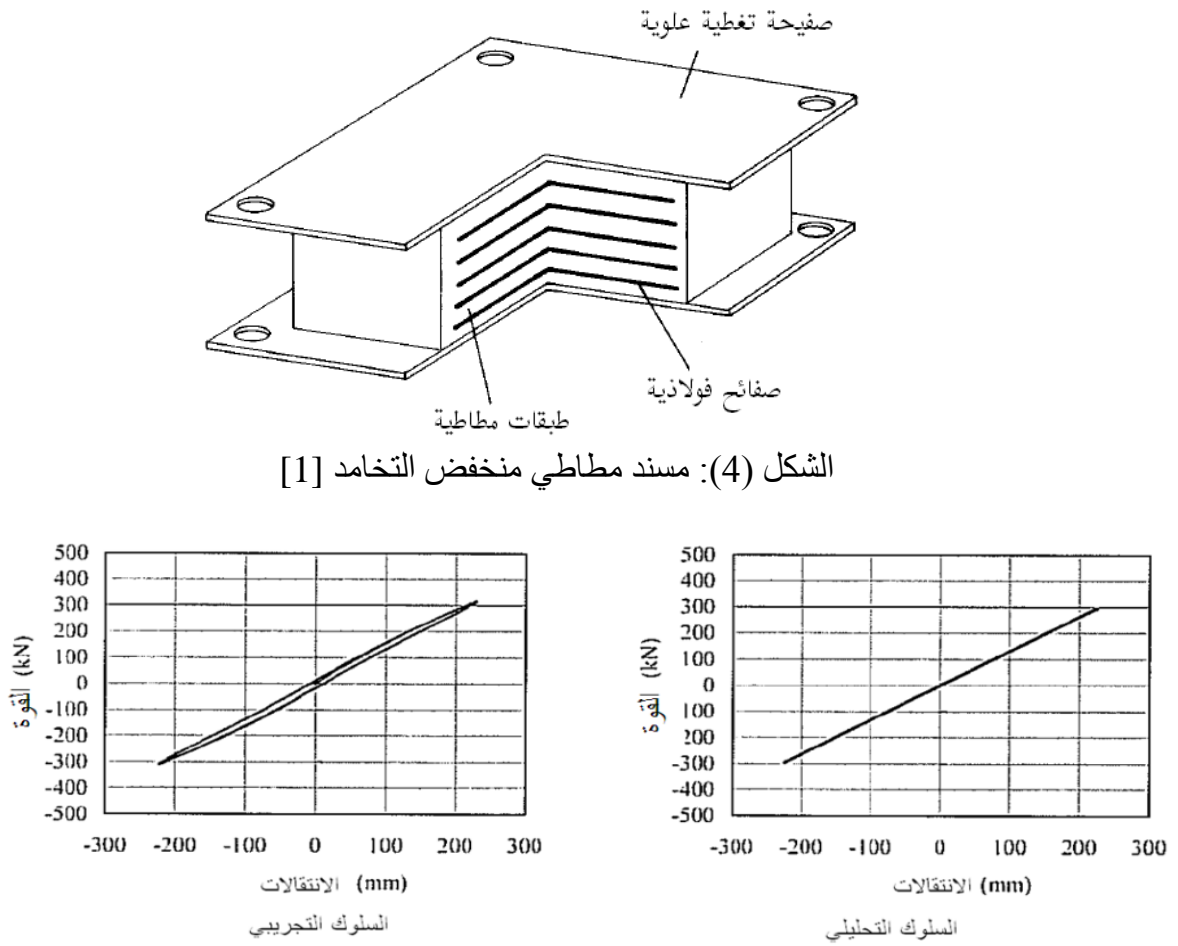

الثكل (5): علاقة القوى-الانتقالات لمسند مطاطي منخفض التخامد [7]

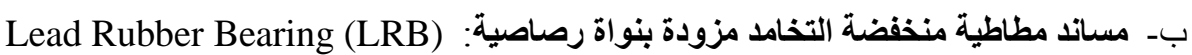

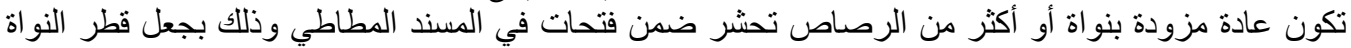

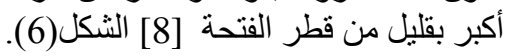
تمتلك النواة الرصاصية القدرة على تبديد الطاقة نتيجة التشو هات اللانة وهي تزود المسند بسلوك ثنائي خطية[8]

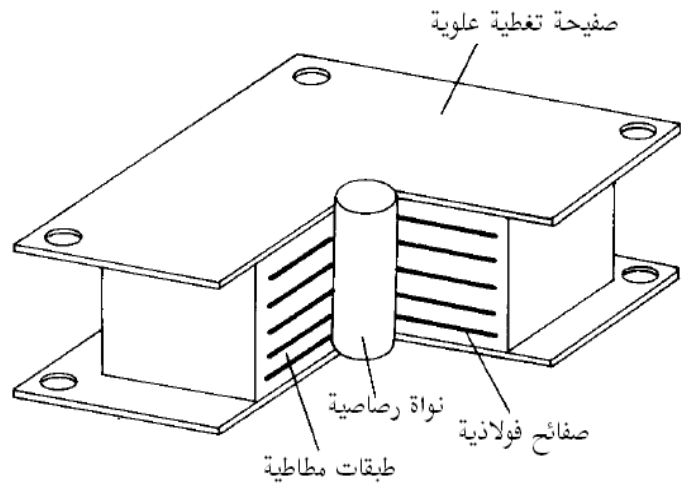

الثكل (6): مسند مطاطي منخفض التخامد مزود بنواة رصاصية [8] 

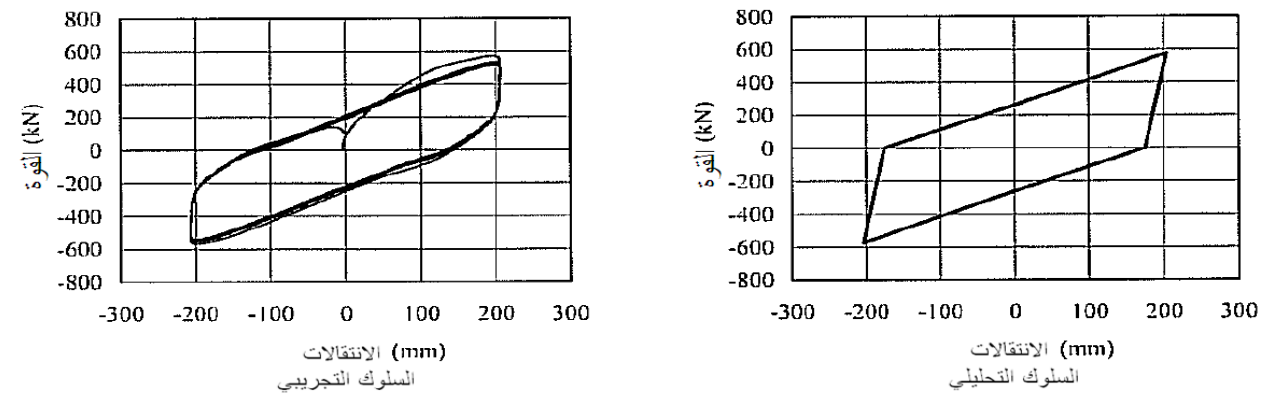

الشكل (7): علاقة القوى-الاجهادات لمسند مطاطي مزود بنواة رصاصية [7]

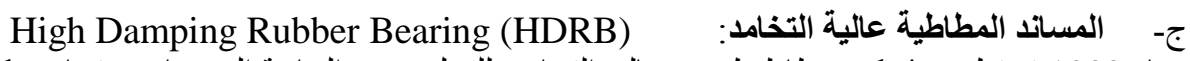

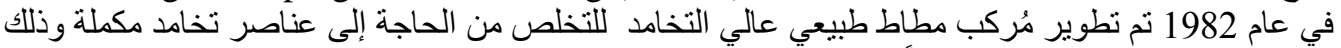

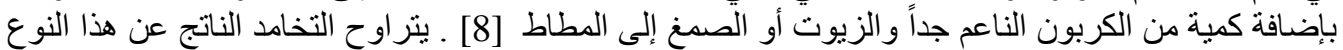

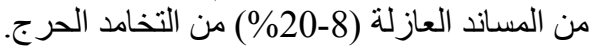
يتمتع هذا المسند بسلوك ثنائي الخطية كما في المساند المطاطية منخفضة التخامد المزودة بنواة رصاصية.

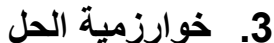

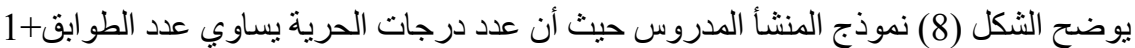

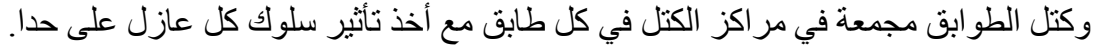

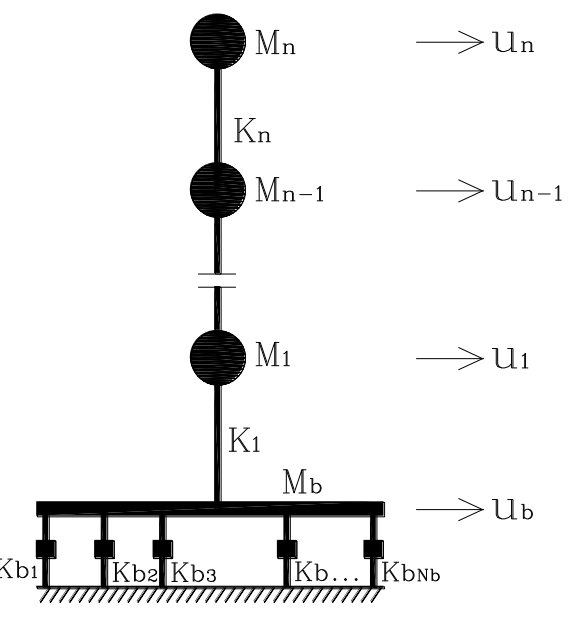

الثكل (8): نموذج (MDOF) لمنشأ معزول زلز اليا

الصلابات الأفقية للمساند العازلة : K

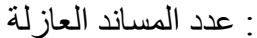

: كتلة الديافر ام فوق مستوى العزل مبانشرة

$\mathrm{N}_{\mathrm{b}}$

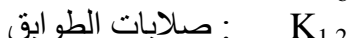

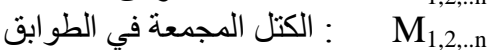

: : عد الطو ابق فوق مستوى العزل : n 
سنقوم بدراسة نموذج معرض لسجل زمني لهزة أرضية” "Time history" بطريقة نيومارك للتكامل المباشر (الخئ تحدد مصفوفة الكتلة [M] على النحو النتالي :

$[M]=\left[\begin{array}{cccc}M b & 0 & \cdot & 0 \\ 0 & M 1 & \cdot & \cdot \\ \cdot & \cdot & \cdot & \cdot \\ 0 & \cdot & \cdot & M n\end{array}\right]$

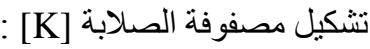
تحديد سلوك المساند العازلة في اللحظة الزمنية t t

كما أنثرنا سابقا أن لكل من هذه المساند المطاطية سلوكاً ثنائي الخطية كما في الثنكل (9) Force

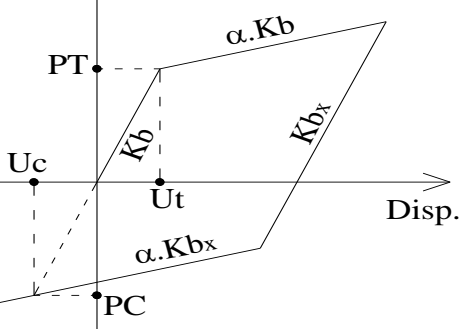

الثكل (9): نمذجة لسلوك العازل ثنائي الخطية

من المكن تجزئة علاقة القوى-الانتقالات السابقة للعازل كما في الثكل(10)

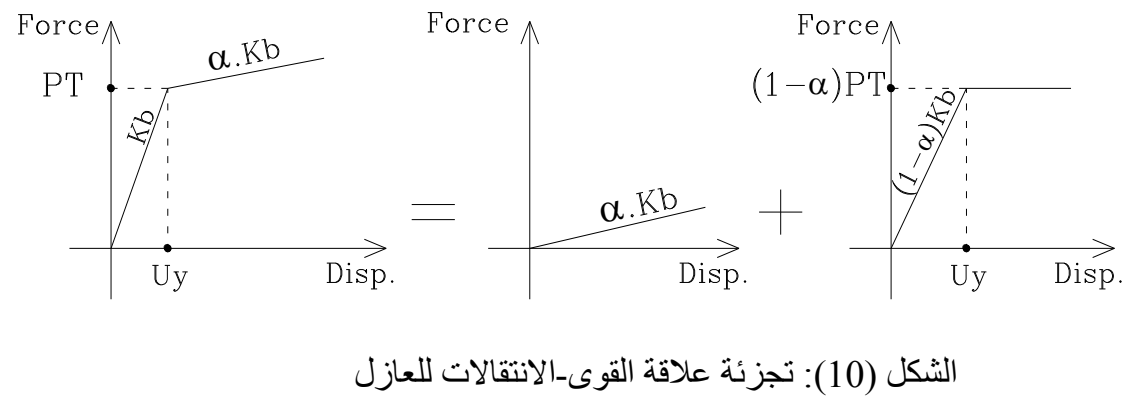

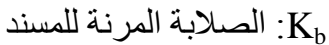
PT

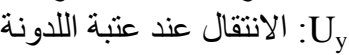
Q 


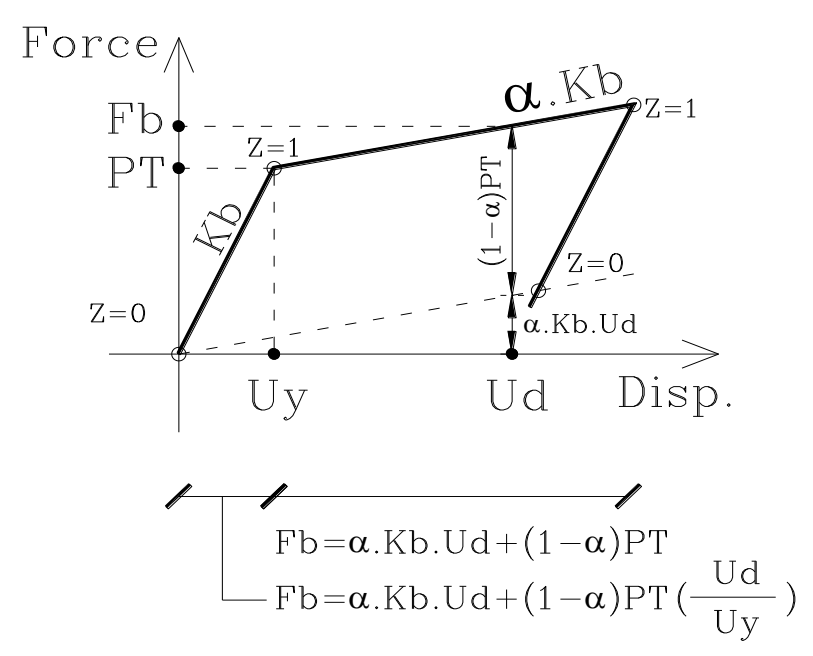

الشكل (11): قوى القص الناشئة ضمن المسند

$\mathrm{Fb}=\alpha * \mathrm{~K}_{\mathrm{b}} * \mathrm{U}_{\mathrm{d}}+(1-\alpha) * \mathrm{PT} *(\mathrm{Ud} / \mathrm{Uy})$

قوة القص الناشئنة ضمن ما يلي: المسند في مرحلة المرونة

$\mathrm{Fb}=\alpha * \mathrm{~K}_{\mathrm{b}} * \mathrm{U}_{\mathrm{d}}+(1-\alpha) * \mathrm{PT}$

قوة القص الناشئة ضمن المسند في مرحلة ما بعد التلدن

$\mathrm{Fb}=\alpha * \mathrm{~K}_{\mathrm{b}} * \mathrm{U}_{\mathrm{d}}+(1-\alpha) * \mathrm{PT} * \mathrm{z}$

يمكن كتابة العلاقتين السابقتين بالثكل :

$$
-1 \leq z=\frac{U_{d}}{U_{y}} \leq 1
$$

حيث :

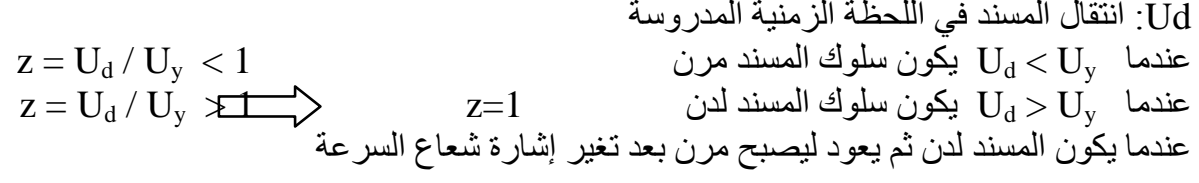

Force

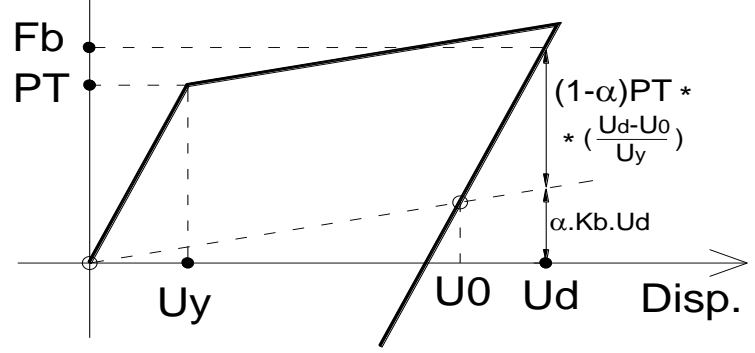

(12) (12) - (الثكل 
$\mathrm{Fb}=\square * \mathrm{~K}_{\mathrm{b}} * \mathrm{U}_{\mathrm{d}}+(1-\square) * \mathrm{PT} *\left(\mathrm{U}_{\mathrm{d}}-\mathrm{U}_{\mathrm{o}} / \mathrm{U}_{\mathrm{y}}\right)$

في هذه الحالة تكون قوة القص الناشئة ضمن المسند

$\mathrm{Fb}=\square * \mathrm{~K}_{\mathrm{b}} * \mathrm{U}_{\mathrm{d}}+(1-\square) * \mathrm{PT} * \mathrm{z}$

$$
-1 \leq z=\frac{U_{d}-U_{0}}{U_{y}} \leq 1
$$

يجب تحديد قيمة جديدة لـ U Uي كل لحظة يكون فيها المنشاً لان ثم يعود ليصبح مرن

بفرض

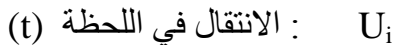

(t) التغير في الانتقال في اللحظة في الالنظة :

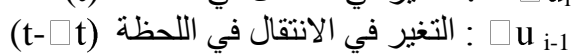

$\mathrm{U}_{0}=\mathrm{U}_{\mathrm{i}}-\mathrm{U}_{\mathrm{y}}$ عندما

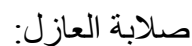

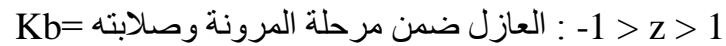

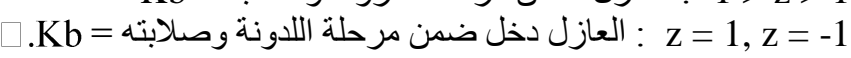

نقوم بتكرار الحلقة السابقة على جميع المساند العازلة لنتمكن من حساب صلابة نظام العزل الزلز الي خلال كل

$$
\begin{aligned}
& K_{b}=\sum_{i=1}^{N_{b}} K_{b i} \\
& \text { Force }_{b}=\sum_{i=1}^{N_{b}} F_{b i} \\
& \text { Force } \\
& {[K]=\left[\begin{array}{ccccc}
K_{b}+K_{1} & -K_{1} & 0 & . & 0 \\
-K_{1} & K_{1}+K_{2} & -K_{2} & 0 & . \\
0 & -K_{2} & \cdot & \cdot & 0 \\
\cdot & 0 & \cdot & K_{n-1}+K_{n} & -K_{n} \\
0 & \cdot & 0 & -K_{n} & K_{n}
\end{array}\right]}
\end{aligned}
$$

وكما بينا سابقاً فإن التخميد الطبيعي ضمن الجملة الإنشائية يكاد يكون مهملاً بالمقارنة مع التخميد الناتج في نظام

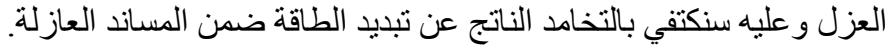

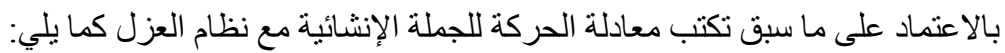

$$
[M] \cdot\{\ddot{U}\}+[K] \cdot\{U\}=\{F e\}
$$

$$
\begin{aligned}
& \{U\}=\left\{\begin{array}{lllll}
U_{b} & U_{1} & U_{2} & \ldots & U_{n}
\end{array}\right\}^{T} \\
& \{\ddot{U}\}=\left\{\begin{array}{lllll}
\ddot{U}_{b} & \ddot{U}_{1} & \ddot{U}_{2} & \ldots & \ddot{U}_{n}
\end{array}\right\}^{T} \\
& \{F \boldsymbol{F}\}=\left\{\begin{array}{lllll}
F \boldsymbol{C}_{b} & F \boldsymbol{C}_{1} & F \boldsymbol{C}_{2} & \ldots & F \boldsymbol{e}_{n}
\end{array}\right\}^{T}
\end{aligned}
$$

باستخدام طريقة نيومارك في التكامل العددي المباشر نكتب: 


$$
\begin{aligned}
\dot{U}^{i+1} & =\dot{U}^{i}+\left\lfloor(1-\delta) \ddot{U}^{i}+\delta \cdot \ddot{U}^{i+1}\right\rfloor \Delta t \\
U^{i+1} & =U^{i}+\dot{U}^{i} . \Delta t+\left[\left(\frac{1}{2}-\alpha\right) \ddot{U}^{i}+\alpha \cdot \ddot{U}^{i+1}\right] \Delta t^{2}
\end{aligned}
$$

يمكن كتابة العلاقتان السابقتان على الثكل التالي :

$$
\begin{aligned}
& \Delta \dot{U}^{i+1}=\left[(1-\delta) \ddot{U}^{i}+\delta \cdot \ddot{U}^{i+1}\right] \Delta t \\
& \Delta U^{i}=\dot{U}^{i} \cdot \Delta t+\left[\left(\frac{1}{2}-\alpha\right) \ddot{U}^{i}+\alpha \cdot \ddot{U}^{i+1}\right] \Delta t^{2}
\end{aligned}
$$

$$
\left[K_{t}\right] \cdot\{\Delta U\}=\{\Delta F e\}
$$

بالتعويض في معادلة الحركة (6q) ينتج:

$$
\left[K_{t}\right]=[K]+\frac{1}{\alpha \cdot \Delta t^{2}}[M]
$$

متجه التغير في القوى

$$
\left[\Delta F e_{i}\right\}=\left\{F e_{i+1}\right\}-\left\{F e_{i}\right\}+\frac{6}{\Delta t}[M]\{\dot{U}\}_{i}+3[M]\{\ddot{U}\}_{i}
$$

$$
\text { متجه التغير في الانتقالات }
$$

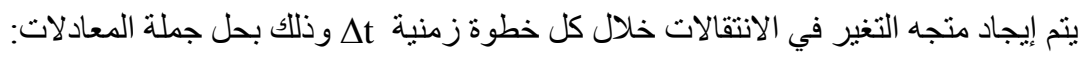

$\left[K_{t}\right] \cdot\{\Delta U i\}=\{\Delta F e i\}$

$\left\{\Delta \dot{U}_{i}\right\}=\frac{3}{\Delta t}\left\{\Delta U_{i}\right\}-3\left\{\dot{U}_{i}\right\}-\frac{\Delta t}{2}\left\{\ddot{U}_{i}\right\}$
$\mathrm{Eq}(10)$

ثم نوجد منجهي الانتقالات و السرعة:

$\left\{\dot{U}_{i+1}\right\}=\left\{\dot{U}_{i}\right\}+\left\{\Delta \dot{U}_{i}\right\} \quad\left\{U_{i+1}\right\}=\left\{U_{i}\right\}+\left\{\Delta U_{i}\right\}$

نوجد منجه القوى المطبقة في جميع الطو ابق

$\{$ Force $\}=[K] .\{U D\}$

$\mathrm{Eq}(12)$

نوجد قيمة التسار ع من العلاقة الأساسية لتوازن المنشأ

$[M] .\{\ddot{U}\}_{i+1}+[K] .\{U\}_{i+1}=\{F e\}_{i+1}$ $\mathrm{Eq}(13)$ 
خطوات الحل العددي:

$\mathrm{Eq}(1)$

1 1M]. تشكيل مصفوفة الكتلة

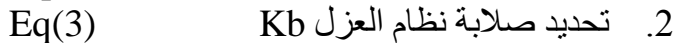

$\mathrm{Eq}(5)$

3.

4.

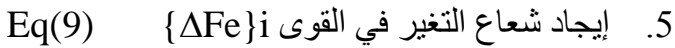

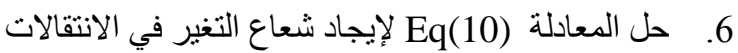

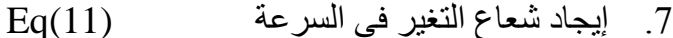

$\mathrm{Eq}(2)$

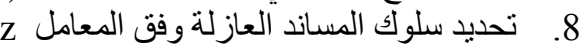

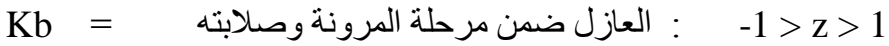

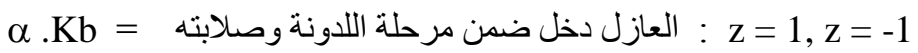

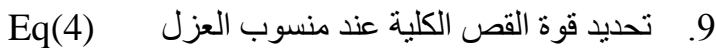

$\mathrm{Eq}(12)$

10. تحديد متجه القوى

$\mathrm{Eq}(13)$

11. تحديد قيمة التسارع الترى

12. العودة إلى الخطوة 2 لتكر ار الخطوات السابقة حتى انتهاء تابع التحريض.

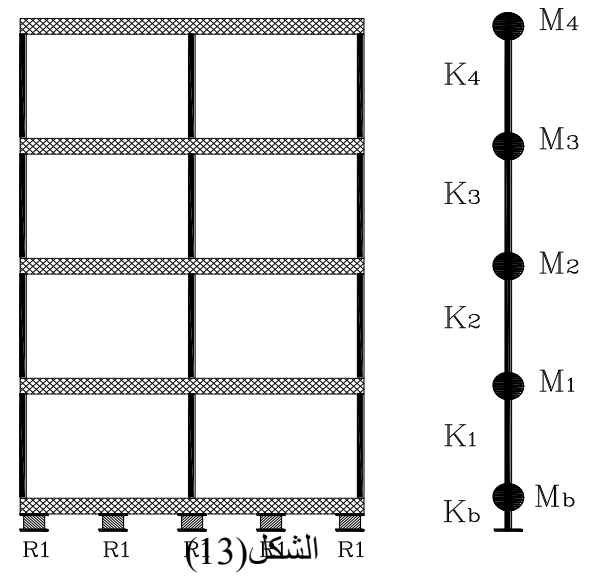

إطار قصي مؤلف من أربعة طو ابق موضح في الثكل(13) الكتل المجمعة في الطو ابق: $\mathrm{Mb}=\mathrm{M} 1=\mathrm{M} 2=\mathrm{M} 3=\mathrm{M} 4=50000 \mathrm{~kg}$ أبعاد مقاطع الأعدة: 50 x $50 \mathrm{~cm}$ E=2.5x10 x E10 N/m²: تابت المرونة

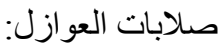
بفرض وجود خمسة مساند اثنتان منها ذات سلوك خطي من

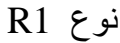

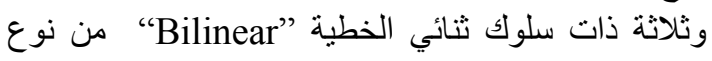

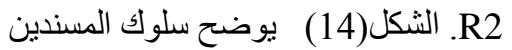

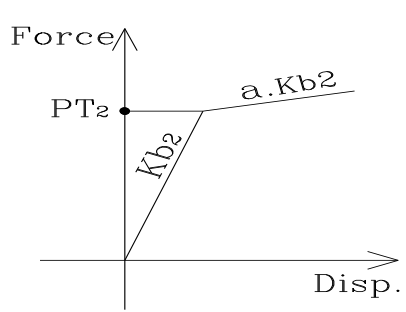

Rubber Isolator (R1)

Rubber Isolator (R2) الثكل(14)

$\mathrm{Kb}_{1}=1.10^{6} \quad \mathrm{~N} / \mathrm{m} \quad \mathrm{Kb}_{2}=5.10^{6} \quad \mathrm{~N} / \mathrm{m}$

$\mathrm{PT}_{2}=1.10^{5} \quad \mathrm{~N} \quad \alpha=0.15$

تم تعريض قاعدة الإطار لهزة Elconting 
تم إيجاد استجابة المنشأ باستخدام برنامج كتب وفق الخوارزمية السابقة بلغة "Visual Basic" ثم باستخدام

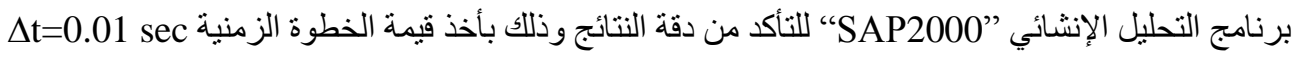
تشكيل مصفوفة كتلة الإطار:

$$
[M]=\left[\begin{array}{ccccc}
M_{b} & 0 & 0 & 0 & 0 \\
0 & M_{1} & 0 & 0 & 0 \\
0 & 0 & M_{2} & 0 & 0 \\
0 & 0 & 0 & M_{3} & 0 \\
0 & 0 & 0 & 0 & M_{4}
\end{array}\right]=\left[\begin{array}{ccccc}
50 & 0 & 0 & 0 & 0 \\
0 & 50 & 0 & 0 & 0 \\
0 & 0 & 50 & 0 & 0 \\
0 & 0 & 0 & 50 & 0 \\
0 & 0 & 0 & 0 & 50
\end{array}\right] .10^{3}
$$

تشكيل مصفوفة صلابة 1لإطار:

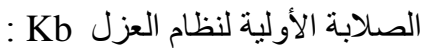

$$
\mathrm{K}_{\mathrm{b}}=\mathrm{K}_{\mathrm{b} 1} * 2+\mathrm{K}_{\mathrm{b} 2} * 3=17.10^{6} \quad \mathrm{~N} / \mathrm{m}
$$

$$
K c=\frac{12 E I}{L^{3}}=\frac{12 E \frac{b h^{3}}{12}}{L^{3}}=\frac{E b h^{3}}{L^{3}}=24414000 \mathrm{~N} / \mathrm{m}
$$

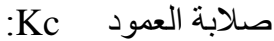

$$
\mathrm{K} 1=\mathrm{K} 2=\mathrm{K} 3=\mathrm{K} 4=3 * \mathrm{Kc}=73242000 \mathrm{~N} / \mathrm{m}
$$

تمت كتابته بلغة "Visual Basic" (الكود البرمجي ضمن الملحق) لحل الخوارزمية واجهة المدخلات مبينة في الشكله(15)

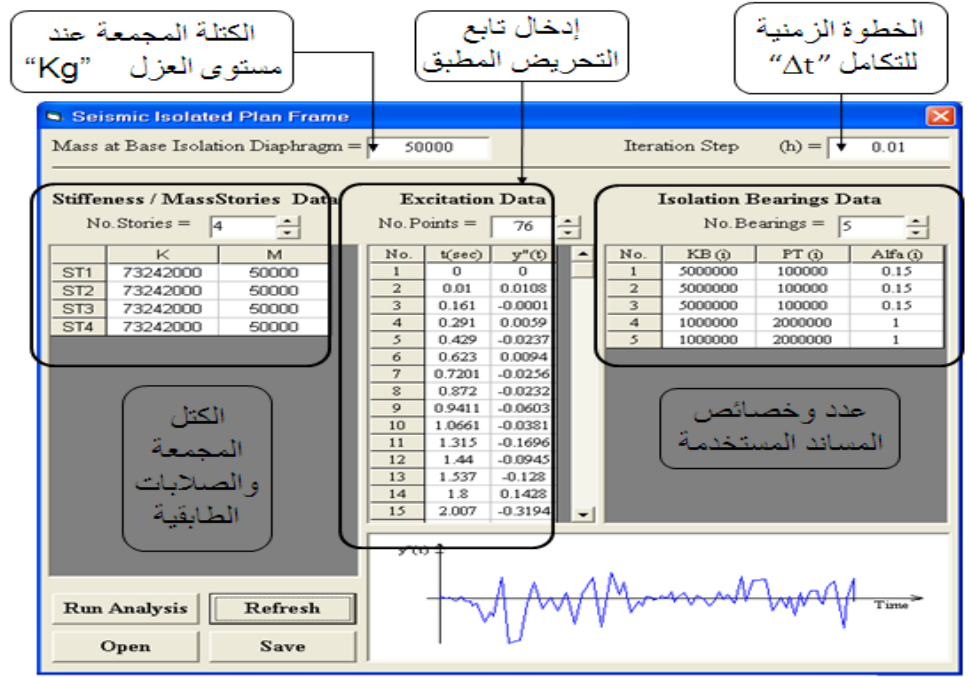

$$
\text { الشكل(15) : واجهة المدخلات للبرنامج }
$$

بالضغط على زر "Run Analysis" يبدأ البرنامج بإجر اء التحليل وتظهر الو اجهة الموضحة في الثكل(16)

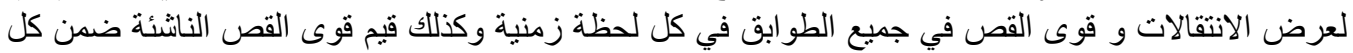

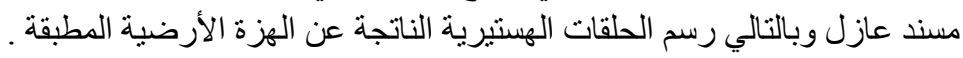




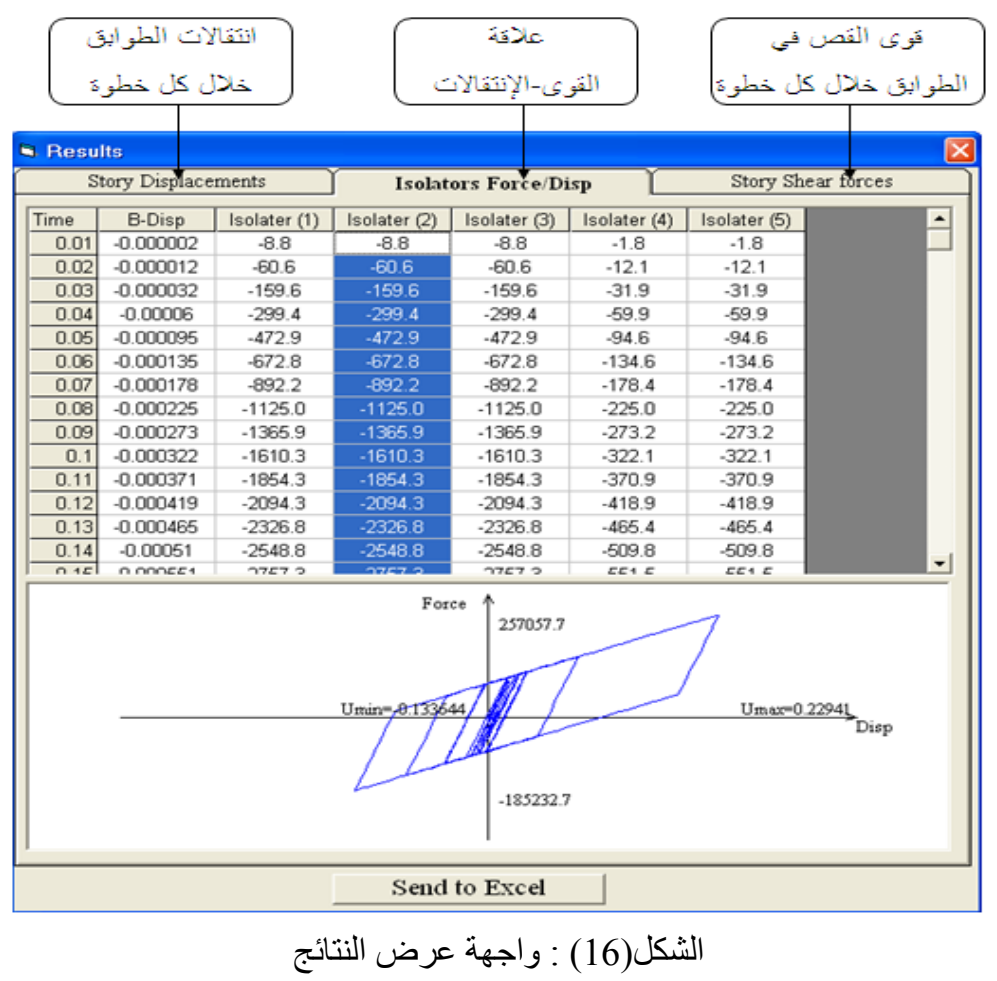

نمذجة الإطار ضمن برنامج”SAP2000" وتعريف خصائص المساند العازلة موضحة في الأشكال(17-18-

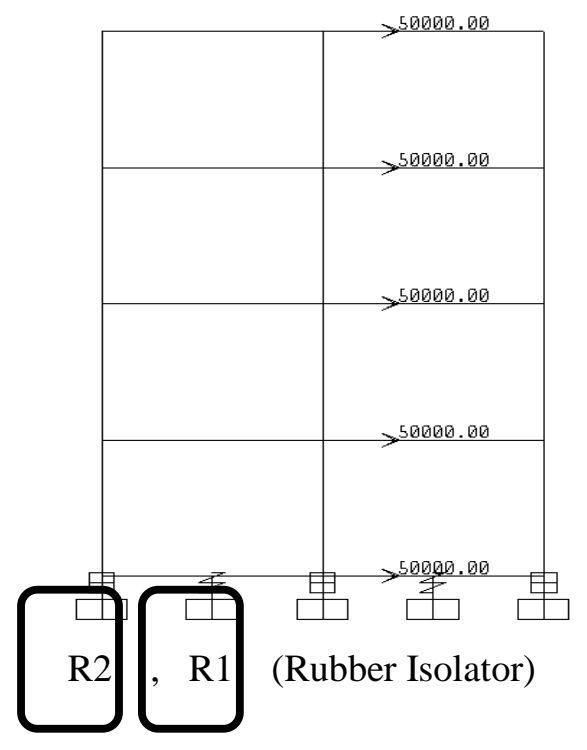

الثكل (17): نمذجة الإطار ضمن برنامج”SAP2000 


\section{Link/Support Directional Properties}

Identification

Property Name

RUB2

Direction

U2

Type

Rubber Isolator

NonLinear

Yes

Properties Used For Linear Analysis Cases

Effective Stiffness

1000000.
0.

Effective Damping

0.

Distance from End J

Properties Used For Nonlinear Analysis Cases

Stiffness

5000000

Yield Strength

100000

Post Yield Stiffness Ratio

0.15

\begin{tabular}{|c|}
\hline$-\ldots K \ldots \ldots . . .$. \\
\hline
\end{tabular}

الثكل(19): خصائص المسند R2"

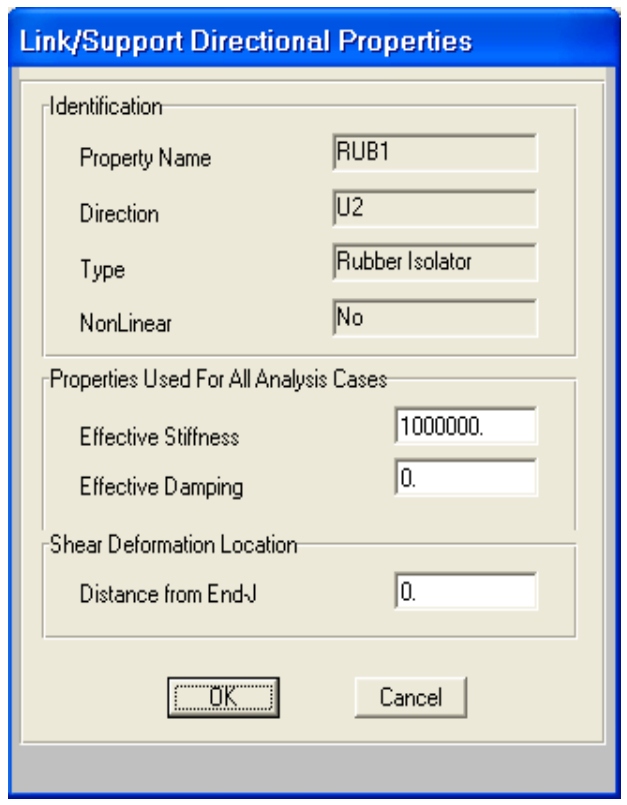

"R1” الثكل(18): خصائص المسند

مقارنة النتائج: يبين الثكل (20) مقارنة بين انتقالات الطابق الأخير الناتجة عن كل من البرنامجين, كما ويبين الثكلين (22, 21) علاقة القوة بالانتقال في المسندين (R1 , R2)

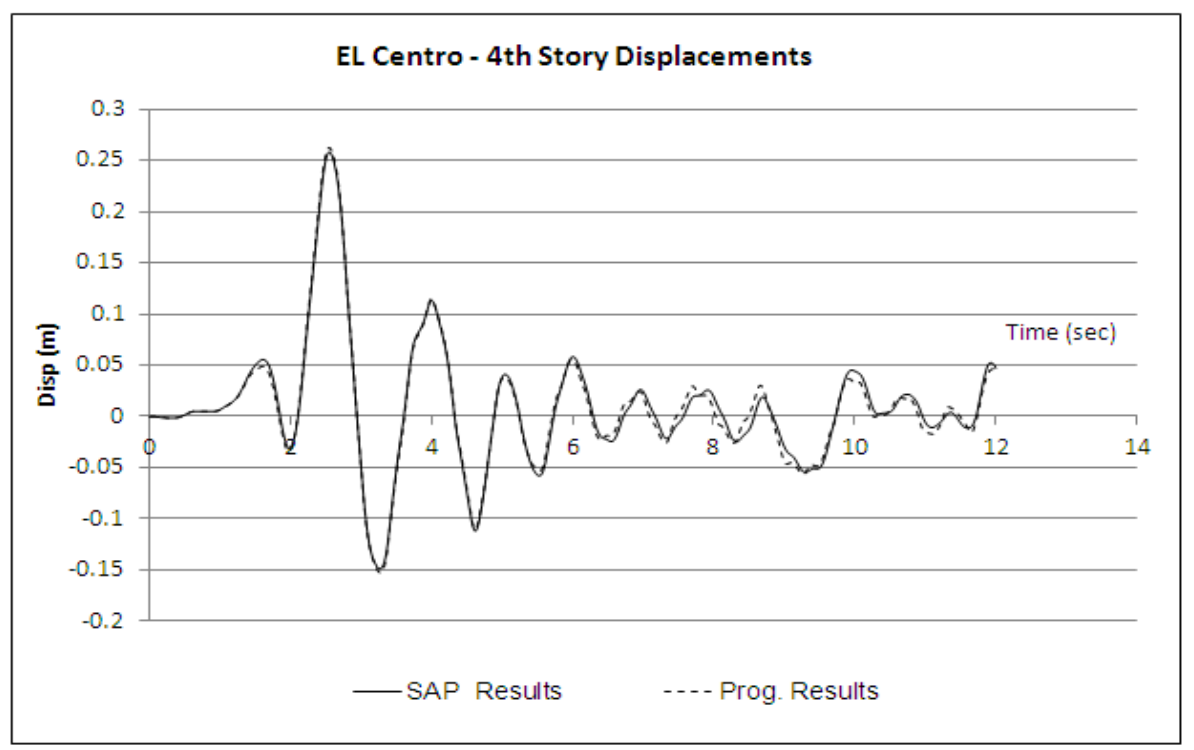

الثكل(20): الانتقالات عند الطابق الرابع 


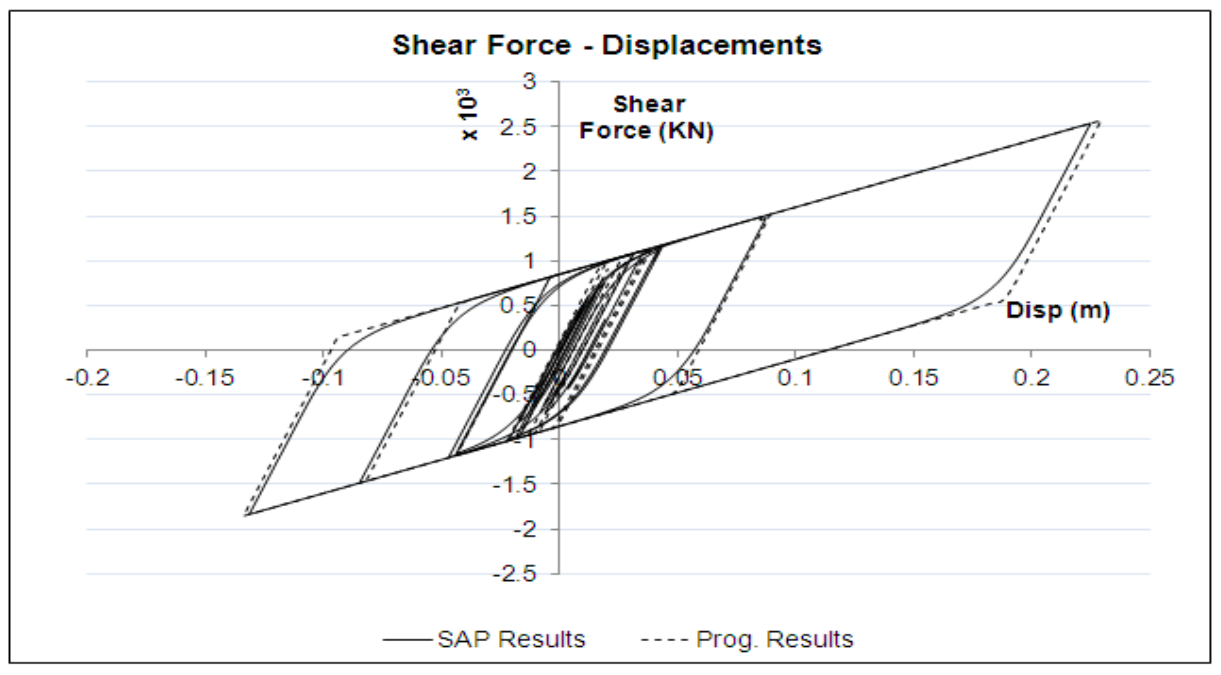

الثكل(21): علاقة (القوى - الانتقالات) للمسند "R2"

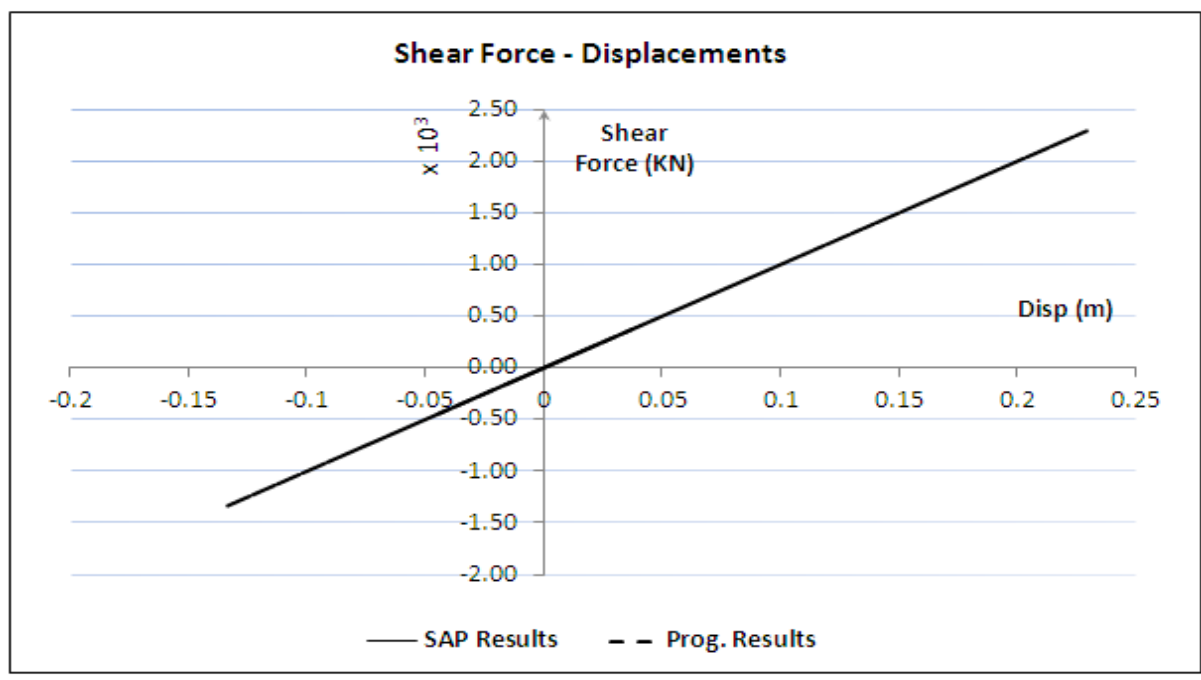

$$
\text { "Ry" الثكل(22): علاقة (القوى - الانتقالات) للمسند }
$$

مقارنة القيم الأعظمية للنتائج : يبين الجدولين (1 2 2$)$ الفرق بين القوى والانتقالات العظمى اللذين تم الحصول

\begin{tabular}{|c|c|c|c|}
\hline $\begin{array}{c}\text { Max. Disp } \\
(\mathrm{cm})\end{array}$ & SAP & Prog. & $\%$ \\
\hline Isolators & 22.50 & 22.90 & 1.8 \\
\hline $4^{\text {th }}$ Story & 25.80 & 26.26 & 1.6 \\
\hline
\end{tabular}

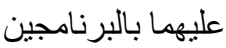

الجدول(2): مقارنة القيم العظمى للانتقالات عند منسوب العزل و الطابق الأخير

\begin{tabular}{|c|c|c|c|}
\hline $\begin{array}{c}\text { Max. Force } \\
(\text { KN })\end{array}$ & SAP & Prog. & $\%$ \\
\hline R1 & 225.00 & 229.50 & 2.0 \\
\hline R2 & 253.70 & 257.10 & 1.3 \\
\hline
\end{tabular}
الجدول (1): مقارنة القيم العظمى

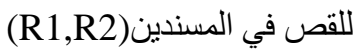

نلاحظ وجود فرق في القيم العظمى ينر اوح بين (1 - 2 \%) 
بالعودة إلى مر اجع برنامج التحليل الإنشائي ”SAP2000" تبين إن سبب الاختلاف في النتائج هو أن السلوك بكوني

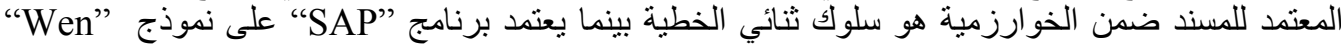

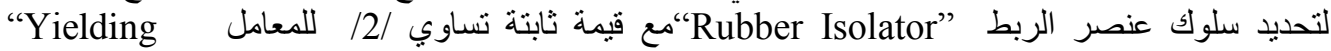

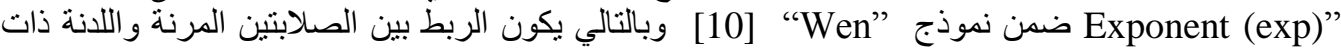

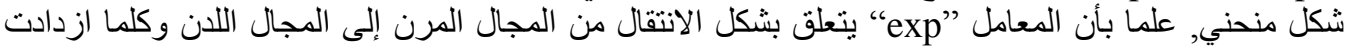

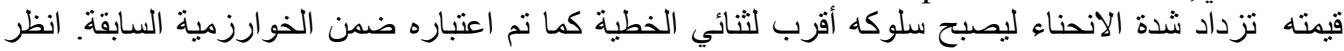
الشكل(23).

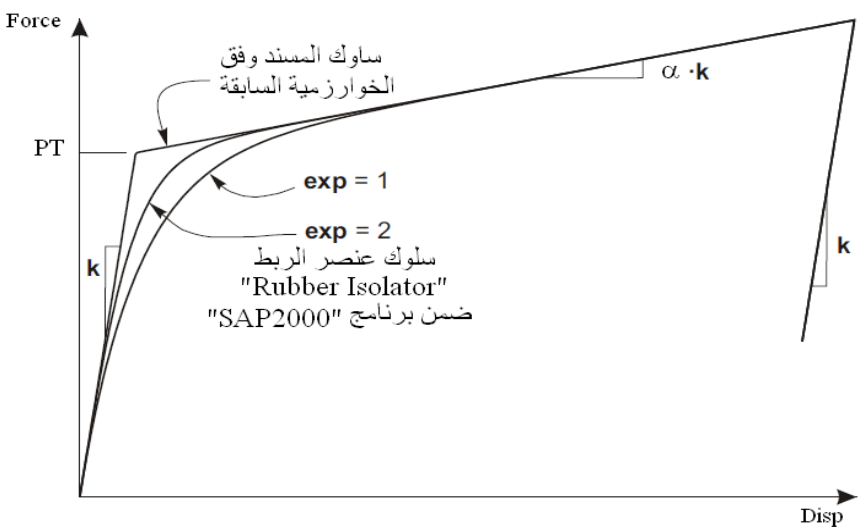

الثكل(23): مقارنة بين سلوك العازل حسب الخو ارزمية وسلو كه ضمن برنامج "SAP"

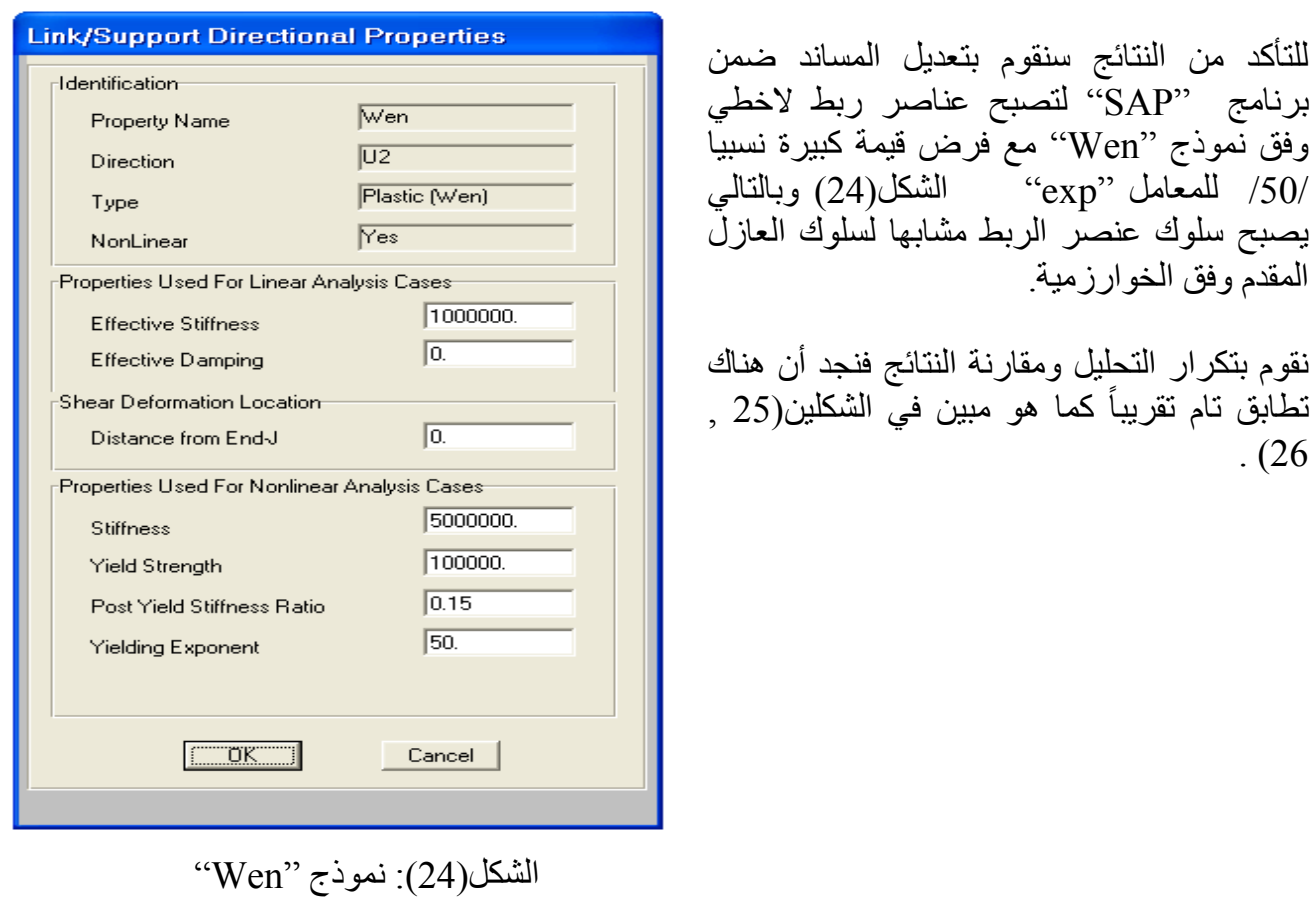




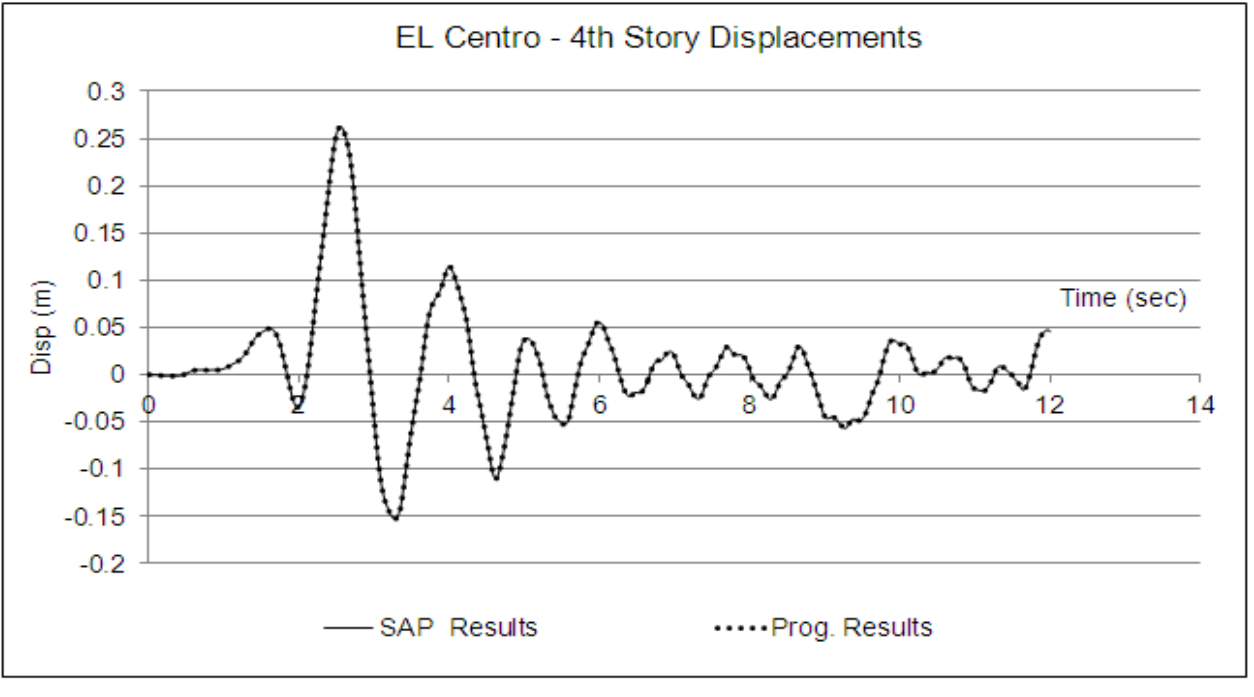

الشكل(25): الانتقالات عند الطابق الرابع

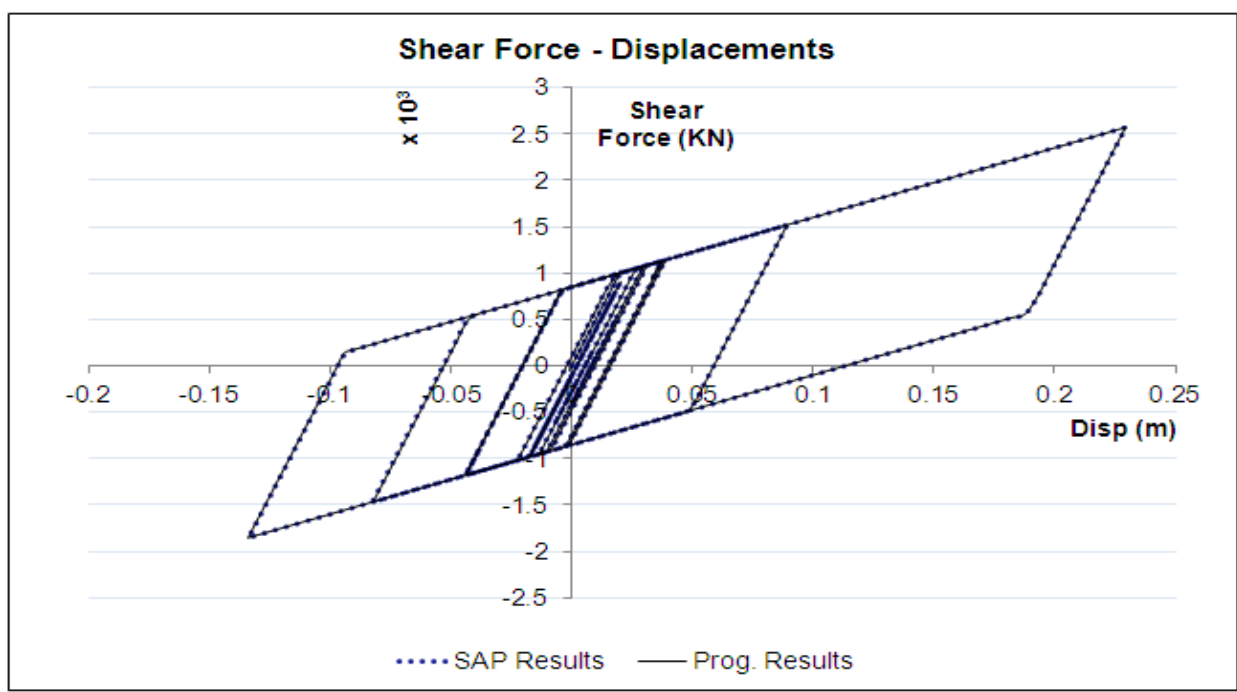

الثكل(26): علاقة (القوى - الانتقالات) للمسند "R2"

كما يتضح من الجدولين (3 , 4) أن الفرق في الانتقالات الأعظمية الناتجة عن البرنامجين هو 0.02\% وكذلك فإن

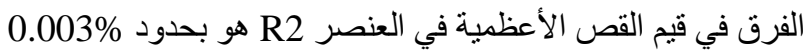

\begin{tabular}{|c|c|c|c|}
\hline $\begin{array}{c}\text { Max. } \\
\text { Disp (cm) }\end{array}$ & SAP & Prog. & $\%$ \\
\hline 4th Story & 26.255 & 26.26 & 0.02 \\
\hline
\end{tabular}

في الطابق الأخير

\begin{tabular}{|c|c|c|c|}
\hline $\begin{array}{l}\text { Max. Force } \\
\text { (KN) }\end{array}$ & SAP & Prog. & $\%$ \\
\hline R2 & 257.136 & 257.144 & 0.003 \\
\hline
\end{tabular}

في المسند (R2) 


\section{6. النتائج :}

في هذا البحث تم عرض خوارزمية لحل المنشآت القصية المعزولة زلز الياً وذللك من خلال اعتبار المنشأ كنموذج

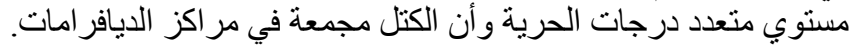
تم اعتبار الجملة الإنشائية فوق نظام العزل ذات سلوك مرن مع أخذ نأثير المساند العازلة كعناصر ذات سلوك ثنائي الخطية.

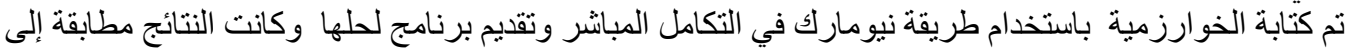

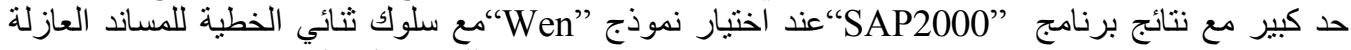

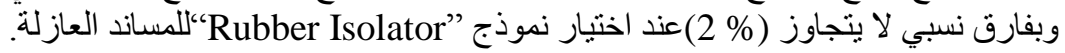

\section{REFERENCES}

1. Master of Science a "Comparison OF Design Specifications for Seismically Isolated Buildings" by EMRE ACAR (2006)

2. Master of Science an "Overview of the Technology and Design of Base Isolated Buildings in High Seismic Regions in the UNITED STATES" by JESSICA IRENE WILES (2008)

3. Kelly, Trever (2001) "Base Isolation of Structures".

4. Masaru Kikuchi, Ian D.Aiken. An Analytical Hysteresis Modal For Elastomeric Seismic Isolation Bearings, Earthquake Engineering and Structural Dynamics, Vol.26, No.2, February (1997).

5. Young-Sang Kim. Study of the effective Stiffness of Base Isolation System for Reducing Acceleration and Displacement Responses, Journal of the Korean Nuclear Society ,Volume 31,No.6,pp.586/594, December(1999).

6. Seval Pinarbasi, Dimitrios Konstantinidis, James M. Kelly. Seismic Isolation for Soft-Story Buildings, 10th World Conference on Seismic Isolation, Energy Dissipation and Active Vibrations Control of Structures, Istanbul, Turkey , May 28-31, 2007.

7. Higashino, Masahiko and Okamoto, Shin (2006) "Response Control and Seismic Isolation of Buildings".

8. Naeim, Farzad and Kelly, James (1999) "Design of Seismic Isolated Structures".

9. Chopra, Anil 2001 "Dynamics of Structures".

10. Computers \& Structures, CSI Analysis Reference Manual, Berkeley, California 